\title{
Efficacy and safety of a new vedolizumab subcutaneous formulation in Japanese patients with moderately to severely active ulcerative colitis
}

\author{
Taku Kobayashi ${ }^{1}$, Hiroaki Ito ${ }^{2}$, Toshifumi Ashida ${ }^{3}$, Tadashi Yokoyama ${ }^{4}$, Masakazu Nagahori ${ }^{5}$, Tomoki Inaba ${ }^{6}$, \\ Mitsuhiro Shikamura ${ }^{7}$, Takayoshi Yamaguchi ${ }^{7}$, Tetsuharu Hori ${ }^{7}$, Philippe Pinton ${ }^{8}$, Mamoru Watanabe ${ }^{5}$, Toshifumi Hibi ${ }^{1}$ \\ ${ }^{1}$ Center for Advanced IBD Research and Treatment, Kitasato University Kitasato Institute Hospital, Tokyo; Infusion clinic, Osaka; \\ ${ }^{3}$ Inflammatory Bowel Disease Center, Sapporo Tokushukai Hospital, Sapporo; ${ }^{4}$ Yokoyama IBD Clinic, Nagoya; ${ }^{5}$ Department of \\ Gastroenterology and Hepatology, Tokyo Medical and Dental University, Tokyo; ${ }^{6}$ Department of Gastroenterology, Kagawa Prefectural Central \\ Hospital, Kagawa; ${ }^{7}$ Takeda Development Center Japan, Takeda Pharmaceutical Company Limited, Osaka; ${ }^{8}$ Japan Medical Office, Takeda \\ Pharmaceutical Company Limited, Tokyo, Japan
}

Background/Aims: A subgroup analysis was conducted in Japanese patients with moderate to severe ulcerative colitis (UC) enrolled in the phase 3 VISIBLE 1 study, which evaluated the safety and efficacy of a new vedolizumab subcutaneous (SC) formulation. Methods: Eligible patients received open-label infusions of vedolizumab $300 \mathrm{mg}$ intravenous (IV) at weeks 0 and 2 in the induction phase. Patients with clinical response by complete Mayo score at week 6 entered the double-blind maintenance phase and were randomized to vedolizumab $108 \mathrm{mg}$ SC every 2 weeks, placebo, or vedolizumab $300 \mathrm{mg}$ IV every 8 weeks. The primary endpoint was clinical remission (complete Mayo score $\leq 2$ points; no individual subscore $>1$ point) at week 52 . Results: Of 49 patients who entered the induction phase, 22 out of 49 patients (45\%) had clinical response at week 6 and were randomized to vedolizumab $108 \mathrm{mg} \mathrm{SC}(\mathrm{n}=10)$, placebo $(\mathrm{n}=10)$, or vedolizumab $300 \mathrm{mg} \mathrm{IV}(\mathrm{n}=2)$. At week 52,4 out of 10 patients (40\%) who received vedolizumab SC had clinical remission versus 2 out of 10 patients (20\%) who received placebo (difference: $20 \%$ [ $95 \%$ confidence interval, -27.9 to 61.8$]$ ). Two patients $(2 / 10,20 \%)$ who received vedolizumab SC experienced an injection-site reaction versus none who received placebo. Conclusions: Our results indicate that the efficacy of vedolizumab SC in a subgroup of Japanese patients with UC are similar with those in the overall VISIBLE 1 study population, and with those established with vedolizumab IV. The safety and tolerability of vedolizumab SC were generally similar to that established for vedolizumab IV. (ClinicalTrials.gov ID NCT0261 1830; EudraCT 2015-000480-14) (Intest Res 2021;19:448-460)

Key Words: Vedolizumab; Ulcerative colitis; Inflammatory bowel disease; Subcutaneous; Maintenance

\section{INTRODUCTION}

Ulcerative colitis (UC) is a chronic, relapsing, inflammatory disorder of the large bowel that is characterized by a lymphocytic infiltration of the colonic mucosa with resulting loss of mucosal architecture. ${ }^{1} \mathrm{UC}$ requires long-term management

Received April 6, 2020. Revised May 19, 2020. Accepted May 22, 2020. Correspondence to Taku Kobayashi, Center for Advanced IBD Research and Treatment, Kitasato University Kitasato Institute Hospital, 5-9-1 Shirokane, Minato-ku, Tokyo 108-8642, Japan. Tel: +81-3-3444-6161, Fax: +81-3-

3444-2530, E-mail: kobataku@insti.kitasato-u.ac.jp for the induction and maintenance of clinical remission, and adherence to medication is an important challenge. ${ }^{2}$

Subcutaneous (SC) administration of biologics is effective, safe, and well-tolerated. In general, SC is preferred by both patients and healthcare providers, and can be self-administered at home. ${ }^{3}$ Currently, 3 of the 5 licensed biologics that are approved for the treatment of inflammatory bowel disease (IBD) in Japan use the SC route of administration. ${ }^{4-8}$ The tumor necrosis factor (TNF) $\alpha$ antagonists adalimumab and golimumab are approved for SC administration for the treatment of UC. ${ }^{6,7}$ The interleukin-12/23 p40 antagonist ustekinumab is approved 
for SC administration as maintenance therapy for refractory moderate to severe UC patients. ${ }^{8}$

Vedolizumab is a humanized, gut-selective immunoglobulin G1 monoclonal antibody that binds to $\alpha 4 \beta 7$ integrin and blocks lymphocyte infiltration to the gut tissue. ${ }^{9,10}$ Vedolizumab administered through intravenous (IV) infusion (vedolizumab IV) is approved in Japan to treat patients with moderate to severe UC or Crohn's disease (CD). ${ }^{4}$ The efficacy and safety of vedolizumab has been evaluated in randomized, placebo-controlled, phase 3 trials in Japan of patients with UC $(\mathrm{n}=292)^{11}$ or $\mathrm{CD}(\mathrm{n}=157){ }^{12}$ Results from both trials showed that vedolizumab IV had: numerically, but not significantly, greater efficacy over placebo as induction therapy (at week 10); was either numerically or significantly superior over placebo as maintenance therapy (at week 60); and was well tolerated in Japanese patients with $\mathrm{UC}^{11}$ or $\mathrm{CD}{ }^{12}$ In contrast, results from the global, pivotal, phase 3 GEMINI 1 study in a larger number of patients with UC $(\mathrm{n}=895)$ demonstrated that vedolizumab IV had significantly greater efficacy over placebo as induction therapy (at week 6: all outcome measures $P<0.001$ or $P=0.001$ ) and maintenance therapy (at week 52: all outcome measures $P<0.001$ or $P=0.001) .{ }^{13}$ Other differences in clinical characteristics have been reported between patients with IBD from Japan and those from other countries. For example, a recent administrative database analysis of Japanese patients with IBD found no increase in the incidence of non-Hodgkin lymphoma attributable to thiopurine treatment. ${ }^{14}$ However, cohort studies in European patients with IBD reported an association between thiopurine treatment and an increased risk of lymphoproliferative disorders or cancer. ${ }^{15,16}$ It remains to be determined whether the efficacy and safety of vedolizumab SC differs between patients with UC from Japanese and global populations.

The efficacy and safety profile of vedolizumab SC as maintenance therapy was evaluated in the phase 3 VISIBLE 1 study of patients with moderate to severe UC who achieved clinical response at week 6 following vedolizumab IV induction therapy (ClinicalTrials.gov NCT02611830; EudraCT 2015-00048014). ${ }^{17}$ Here, we report results from the Japanese subgroup of patients with UC who were enrolled in VISIBLE 1.

\section{METHODS}

\section{Study Design, Randomization, and Blinding}

This was a subgroup analysis of the Japanese patients who were enrolled in the VISIBLE 1 study, which was a pivotal, phase 3, multicenter, multinational, randomized, double- blind, double-dummy, placebo-controlled trial. ${ }^{17}$ This subgroup analysis evaluated the efficacy and safety of maintenance treatment with vedolizumab SC in adult patients with moderate to severe UC who achieved a clinical response at week 6 with open-label therapy with vedolizumab IV $300 \mathrm{mg}$ at weeks 0 and 2. A vedolizumab IV reference arm was included for descriptive comparisons of efficacy, safety, and immunogenicity with vedolizumab SC.

In the induction phase, eligible patients were enrolled at week 0 , received open-label infusions of vedolizumab IV $300 \mathrm{mg}$ at weeks 0 and 2, and were assessed for clinical response by complete Mayo score at week 6 (defined as a reduction in complete Mayo score $\geq 3$ points and $\geq 30 \%$ from week 0 , with an accompanying decrease in rectal bleeding subscore $\geq 1$ point or absolute rectal bleeding subscore $\leq 1$ point). Patients with clinical response entered the maintenance phase and those with no clinical response received a third IV infusion of vedolizumab $300 \mathrm{mg}$ at week 6 . Patients with clinical response by partial Mayo score at week 14 (defined as a reduction in partial Mayo score $\geq 2$ points and $\geq 25 \%$ from week 0 , with an accompanying decrease in rectal bleeding subscore $\geq 1$ point or absolute rectal bleeding subscore $\leq 1$ point) were eligible to enroll in an open-label extension study (NCT02620046; EudraCT 2015-000482-31). Patients with no clinical response were discontinued.

Patients with clinical response at week 6 were enrolled in the maintenance phase. Patients were randomized in a doubleblind, double-dummy fashion to receive SC injections every 2 weeks (Q2W) and IV infusions every 8 weeks (Q8W), from week 6 through week 50, as follows: vedolizumab SC injections $108 \mathrm{mg}$ Q2W and placebo IV infusions Q8W; or placebo SC injections Q2W and placebo IV infusions Q8W; or vedolizumab IV infusions $300 \mathrm{mg}$ Q8W and placebo SC injections Q2W. SC prefilled syringes were used. Further details on SC injection technique are provided in the Supplementary Material.

Randomization was stratified by concomitant use of oral corticosteroids, clinical remission status at week 6 , and previous failure with TNF- $\alpha$ antagonist treatments or use of a concomitant immunomodulator (azathioprine or 6-mercaptopurine).

Further details on randomization and blinding are provided in the Supplementary Material.

\section{Patients}

Eligible patients were aged 18-80 years and were diagnosed with UC $\geq 6$ months before study enrollment. Patients had moderate to severe UC, defined as complete Mayo score 6-12 
with an endoscopic subscore $\geq 2$; and had inadequate response to, loss of response to, or intolerance to immunomodulators (azathioprine or 6 mercaptopurine), TNF- $\alpha$ antagonists, or corticosteroids. At week 6, patients who received oral corticosteroids and achieved clinical response began a corticosteroid tapering regimen (further details in Supplementary Material). The institutional review board of each study site reviewed and approved the study protocol. This study was conducted in compliance with all institutional review board regulations and in accordance with the ethical principles that have their origin in the Declaration of Helsinki. Informed consent was provided by all patients. For patients aged $<20$ years, additional consent from parents or guardians was required.

\section{Endpoints}

\section{1) Efficacy}

Efficacy analyses for the induction phase (week 6) were conducted in patients enrolled at week 0 . Efficacy analyses for the maintenance phase (week 52) were conducted in patients enrolled at week 0 who had clinical response at week 6 .

The primary efficacy endpoint was clinical remission (defined as complete Mayo score $\leq 2$ points and no individual subscore $>1$ point) at week 52 . Secondary efficacy endpoints included: endoscopic improvement (referred to as mucosal healing in the study protocol) at week 52, defined as Mayo endoscopic subscore of $\leq 1$ point; durable clinical response (defined as clinical response at week 6 and week 52); durable clinical remission (defined as clinical remission at week 6 and week 52); and corticosteroid-free remission (defined as discontinuation of oral corticosteroids, with subsequent clinical remission at week 52, in patients receiving oral corticosteroids at baseline). Clinical remission at week 52 by prior TNF- $\alpha$ antagonist therapy was an exploratory endpoint. Further details on assessments are provided in the Supplementary Material.

\section{2) Patient-Reported Outcomes}

Inflammatory Bowel Disease Questionnaire (IBDQ) total score and subscores, Euro Quality of Life-5DE (Q-5D) visual analog scale (VAS) score, and Work Productivity and Activity Impairment (WPAI-UC) instrument scores were assessed. Further details on patient-reported outcomes (PROs) are provided in the Supplementary Material.

\section{3) Safety/Tolerability}

Adverse events (AEs), defined as any AE regardless of relationship to study drug, were captured during study visits and from any spontaneous reports at any time during the study, and were coded using the Medical Dictionary for Regulatory Activities (MedDRA; version 21.0).

\section{4) Immunogenicity}

The rates of anti-vedolizumab antibody (AVA) development were evaluated using a validated electrochemiluminescence assay. ${ }^{18}$ Positive AVA status was defined as $\geq 1$ positive AVA result from predose to week 52 . Persistently positive AVA status was defined as having a serum sample positive for AVA at $\geq 2$ consecutive visits.

\section{5) Statistical Analysis}

This is an ad-hoc analysis of the Japanese subgroup of patients with UC enrolled in the VISIBLE 1 study. The majority of statistical methods are the same as those described in the VISIBLE 1 study. ${ }^{17}$ However, statistical tests were not performed owing to the lower number of Japanese patients with UC in this ad-hoc analysis.

\section{RESULTS}

\section{Patient Disposition and Baseline Characteristics}

Demographic and baseline characteristics, including racial background, of patients enrolled in the VISIBLE 1 study are shown in Supplementary Table 1. Of the 60 patients screened across 24 sites in Japan, 49 were eligible to enter the induction phase from April 2016 to April 2017 (Fig. 1). At week 6, 22 out of 49 patients (45\%) had a clinical response and were eligible for randomization to receive the following treatments in the maintenance phase: vedolizumab SC $108 \mathrm{mg}(\mathrm{n}=10)$, placebo $(\mathrm{n}=10)$, or vedolizumab IV $300 \mathrm{mg}(\mathrm{n}=2)$.

Baseline characteristics for patients enrolled in the maintenance phase were generally similar to those for patients in the induction phase (Table 1). The mean duration of UC was $7.1 \pm 4.0$ years in the vedolizumab SC group, $10.3 \pm 6.6$ years in the placebo group, and $8.2 \pm 8.0$ years in the vedolizumab IV group (Table 1). Prior failure of TNF- $\alpha$ antagonist therapy had occurred in 5 out of 10 patients $(50 \%)$ in the vedolizumab SC group, 2 out of 10 patients (20\%) in the placebo group, and 2 out of 2 patients (100\%) in the vedolizumab IV group.

\section{Efficacy}

A numerically higher rate of clinical remission was observed at week 52 in the vedolizumab SC group $(4 / 10,40 \%)$ than in the placebo group $(2 / 10,20 \%)$ (difference between groups, $20 \%$ 
Table 1. Demographic and Baseline Characteristics (SAS-Combined) and UC-Related Disease Characteristics (FAS) of Patients Enrolled in the Maintenance Phase

\begin{tabular}{|c|c|c|c|}
\hline Characteristic & $\begin{array}{l}\text { Placebo } \\
(n=10)\end{array}$ & $\begin{array}{l}\text { Vedolizumab SC } \\
\qquad(n=10)\end{array}$ & $\begin{array}{c}\text { Vedolizumab IV } \\
\quad(n=2)\end{array}$ \\
\hline Age (yr) & $43.6 \pm 13.0$ & $46.0 \pm 15.5$ & $54.5 \pm 19.1$ \\
\hline Weight (kg) & $58.2 \pm 10.6$ & $62.9 \pm 16.3$ & $61.8 \pm 6.8$ \\
\hline \multicolumn{4}{|l|}{ Smoking classification } \\
\hline Current smoker & 0 & $2(20.0)$ & 0 \\
\hline Ex-smoker & $6(60.0)$ & $4(40.0)$ & $1(50.0)$ \\
\hline Duration of UC (yr) & $10.3 \pm 6.6$ & $7.1 \pm 4.0$ & $8.2 \pm 8.0$ \\
\hline Prior TNF- $\alpha$ antagonist therapy & $2(20.0)$ & $5(50.0)$ & $2(100.0)$ \\
\hline Concomitant immunomodulator use only at wk 0 & $1(10.0)$ & 0 & 0 \\
\hline \multicolumn{4}{|l|}{ Disease localization } \\
\hline Left-sided colitis & $3(30.0)$ & $4(40.0)$ & $1(50.0)$ \\
\hline Pancolitis & $6(60.0)$ & $5(50.0)$ & $1(50.0)$ \\
\hline \multicolumn{4}{|l|}{ Fecal calprotectin $(\mu \mathrm{g} / \mathrm{g})^{a}$} \\
\hline$\leq 250$ & $1(10.0)$ & 0 & 0 \\
\hline$>250$ to $\leq 500$ & $1(10.0)$ & 0 & 0 \\
\hline$>500$ & $8(80.0)$ & $10(100.0)$ & $2(100.0)$ \\
\hline \multicolumn{4}{|l|}{ Mayo score } \\
\hline Mild (Mayo score <6) & 0 & 0 & 0 \\
\hline
\end{tabular}

Value are presented as mean \pm standard deviation or number (\%).

a Median (minimum-maximum).

SAS, safety analysis set; UC, ulcerative colitis; FAS, full analysis set; SC, subcutaneous; IV, intravenous; TNF, tumor necrosis factor.

[95\% confidence interavl (CI), -27.9 to 61.8]) (Table 2). In the vedolizumab IV group, 1 out of 2 patients (50\%) had clinical remission at week 52 .

The same rates and trend were observed for the proportion of patients with endoscopic improvement at week 52 in all treatment groups (vedolizumab SC group, $40 \%$; placebo group, 20\%; and vedolizumab IV group, 50\%) (Table 2). A higher proportion of patients with durable clinical response was observed at week 52 in the vedolizumab SC group $(8 / 10,80 \%)$ than the placebo group (2/10, 20\%) (difference between groups, $60.0 \%$ [95\% CI, 12.7 to 88.5]) (Table 2). In the vedolizumab IV group, 1 out of 2 patients (50\%) had durable clinical response at week 52. A numerically greater proportion of patients with durable clini- cal remission was observed at week 52 in the vedolizumab SC group $(2 / 10,20 \%)$ than the placebo group $(1 / 10,10 \%)$ (Table 2$)$. Corticosteroid-free remission at week 52 was observed in 1 out of 4 (25\%) of patients in the vedolizumab SC group and 1 out of $3(33 \%)$ of patients in the placebo group (Table 2). No patients in the vedolizumab IV group had durable clinical remission at week 52 .

In patients who failed prior TNF- $\alpha$ antagonist therapy, clinical remission at week 52 was observed in 1 out of 5 patients $(20 \%)$ in the vedolizumab SC group and in 0 out of 2 patients in the placebo group (difference between groups, 20.0\% [95\% CI, -65.4 to 84.1]) (Table 3). In patients who were TNF- $\alpha$ antagonist therapy naïve, clinical remission at week 52 was ob- 


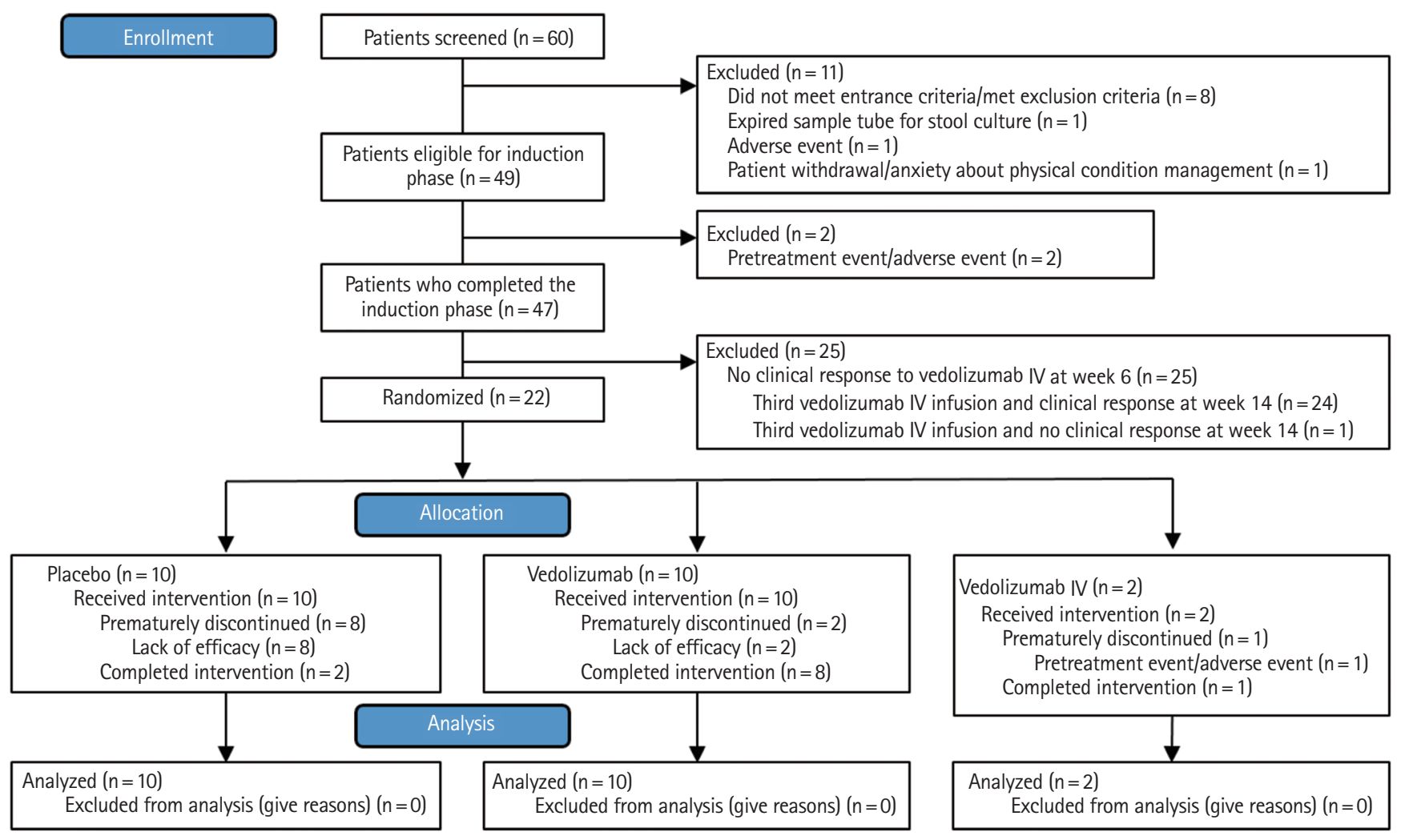

Fig. 1. Patient disposition diagram. IV, intravenous.

served in 3 out of 5 patients (60\%) in the vedolizumab SC group and 2 out of 8 patients (25\%) in the placebo group (difference between groups, 35.0\% [95\% CI, -23.4 to 78.9]) (Table 3).

A summary of fecal calprotectin levels by study visit is shown in Supplementary Table 2.

\section{Patient-Reported Outcomes}

In all patients, IBDQ and EQ-5D VAS PRO instrument scores increased by week 6 following open-label, IV induction therapy with vedolizumab at weeks 0 and 2 (Supplementary Figs. 1, 2). During maintenance treatment, IBDQ and EQ-5D VAS scores generally decreased for patients receiving placebo, while patients receiving vedolizumab SC and IV maintained their improvement in scores following induction therapy (Supplementary Figs 1, 2). In all patients, WPAI-UC instrument scores decreased or stayed the same by week 6 following open-label vedolizumab IV induction at weeks 0 and 2 (Supplementary Fig. 3). During maintenance treatment, WPAI-UC scores increased for patients on placebo, while patients on vedolizumab SC and IV maintained the improvement in scores they had achieved following induction treatment (Supplementary Fig. 3).

\section{Safety}

In the induction phase, 2 patients discontinued treatment with vedolizumab IV $300 \mathrm{mg}$ due to a pretreatment event or AE. In the maintenance phase, planned study drug injections were completed by 8 out of 10 patients $(80 \%)$ in the vedolizumab SC group and 2 out of 10 patients $(20 \%)$ in the placebo group; and planned infusions were completed by 1 out of 2 patients $(50 \%)$ in the vedolizumab IV group. The primary reason(s) for discontinuation was lack of efficacy in the vedolizumab SC and placebo groups, and pretreatment event or AE in the vedolizumab IV group.

In the induction phase, treatment-emergent AEs (TEAEs) were reported in 20 out of 27 patients (74\%) (Table 4). The most frequent TEAE was nasopharyngitis (Table 5). Drug-related TEAEs occurred in 1 out of 27 patients (4\%) (Table 4). Most AEs were mild to moderate and no deaths occurred. Serious TEAEs occurred in 3 out of 27 patients (11\%). TEAEs leading to discontinuation occurred in 2 out of 27 patients (7\%). There were no injection-site reactions (Table 5).

In the maintenance phase, TEAEs were reported in 21 out of 22 patients (96\%), as follows: vedolizumab SC, 9 out of 10 patients (90\%); placebo, 10 out of 10 patients (100\%); and 
Table 2. Proportion of Patients with Clinical Remission, Endoscopic Improvement, Durable Clinical Remission, Durable Clinical Response, and Corticosteroid-Free Remission (FAS)

\begin{tabular}{|c|c|c|c|c|c|c|}
\hline \multirow{2}{*}{ Outcome } & \multicolumn{2}{|c|}{ Placebo $(n=10)$} & \multicolumn{2}{|c|}{ Vedolizumab SC $(n=10)$} & \multicolumn{2}{|c|}{ Vedolizumab IV ( $n=2)$} \\
\hline & No. (\%) & $95 \% \mathrm{Cl}$ & No. (\%) & $95 \% \mathrm{Cl}$ & No. (\%) & $95 \% \mathrm{Cl}$ \\
\hline \multicolumn{7}{|l|}{ Clinical remission at week 52} \\
\hline No & $8(80.0)$ & $(44.4,97.5)$ & $6(60.0)$ & $(26.2,87.8)$ & $1(50.0)$ & $(1.3,98.7)$ \\
\hline Vedolizumab vs. placebo & - & - & $20.0^{\mathrm{a}}$ & $(-27.9,61.8)$ & $30.0^{\mathrm{a}}$ & $(-52.5,86.0)$ \\
\hline Yes & $6(60.0)$ & $(26.2,87.8)$ & $4(40.0)$ & $(12.2,73.8)$ & 0 & \\
\hline No & $4(40.0)$ & $(12.2,73.8)$ & $6(60.0)$ & $(26.2,87.8)$ & $2(100.0)$ & $(15.8,100.0)$ \\
\hline \multicolumn{7}{|c|}{ Endoscopic improvement at week 52} \\
\hline Yes & $2(20.0)$ & $(2.5,55.6)$ & $4(40.0)$ & $(12.2,73.8)$ & $1(50.0)$ & $(1.3,98.7)$ \\
\hline Yes & $2(20.0)$ & $(2.5,55.6)$ & $8(80.0)$ & $(44.4,97.5)$ & $1(50.0)$ & $(1.3,98.7)$ \\
\hline No & $8(80.0)$ & $(44.4,97.5)$ & $2(20.0)$ & $(2.5,55.6)$ & $1(50.0)$ & $(1.3,98.7)$ \\
\hline Vedolizumab vs. placebo & - & - & $60.0^{\mathrm{a}}$ & $(12.7,88.5)$ & $30.0^{\mathrm{a}}$ & $(-52.5,86.0)$ \\
\hline \multicolumn{7}{|c|}{ Durable clinical remission at week 6 and week 52} \\
\hline Yes & $1(10.0)$ & $(0.3,44.5)$ & $2(20.0)$ & $(2.5,55.6)$ & 0 & \\
\hline No & $9(90.0)$ & $(55.5,99.7)$ & $8(80.0)$ & $(44.4,97.5)$ & $2(100.0)$ & $(15.8,100.0)$ \\
\hline Vedolizumab vs. placebo & - & - & $10.0^{\mathrm{a}}$ & $(-36.9,53.9)$ & $-10.0^{\mathrm{a}}$ & $(-84.2,71.0)$ \\
\hline \multicolumn{7}{|c|}{ Corticosteroid-free remission at week 52} \\
\hline No. of patients & 3 & & 4 & & 0 & \\
\hline
\end{tabular}

${ }^{a}$ Risk difference (\%).

FAS, full analysis set; SC, subcutaneous; IV, intravenous; $\mathrm{Cl}$, confidence interval.

Table 3. Proportion of Patients with Clinical Remission at Week 52 by Prior TNF- $\alpha$ Antagonist Therapy (FAS)

\begin{tabular}{|c|c|c|c|c|c|c|}
\hline \multirow{2}{*}{ Prior TNF- $\alpha$ antagonist therapy } & \multicolumn{2}{|c|}{ Placebo $(n=10)$} & \multicolumn{2}{|c|}{ Vedolizumab SC $(n=10)$} & \multicolumn{2}{|c|}{ Vedolizumab IV $(n=2)$} \\
\hline & No. (\%) & $95 \% \mathrm{Cl}$ & No. (\%) & $95 \% \mathrm{Cl}$ & No. (\%) & $95 \% \mathrm{Cl}$ \\
\hline Prior TNF- $\alpha$ antagonist therapy & 2 & & 5 & & 2 & \\
\hline Yes & 0 & & $1(20.0)$ & $(0.5,71.6)$ & $1(50.0)$ & $(1.3,98.7)$ \\
\hline No & $2(100.0)$ & $(15.8,100.0)$ & $4(80.0)$ & $(28.4,99.5)$ & $1(50.0)$ & $(1.3,98.7)$ \\
\hline TNF- $\alpha$ antagonist therapy naïve & 8 & & 5 & & 0 & \\
\hline Yes & $2(25.0)$ & $(3.2,65.1)$ & $3(60.0)$ & $(14.7,94.7)$ & 0 & \\
\hline No & $6(75.0)$ & $(34.9,96.8)$ & $2(40.0)$ & $(5.3,85.3)$ & 0 & \\
\hline Vedolizumab vs. placebo & - & - & $35.0^{a}$ & $(-23.4,78.9)$ & - & - \\
\hline
\end{tabular}

${ }^{a}$ Risk difference (\%).

TNF- $\alpha$, tumor necrosis factor $\alpha$; FAS, full analysis set; SC, subcutaneous; IV, intravenous; Cl, confidence interval.

vedolizumab IV, 2 out of 2 patients (100\%) (Table 4$)$. The most frequent TEAE was nasopharyngitis (Table 5). Drug-related
TEAEs were as follows: vedolizumab SC, 3 out of 10 patients (30\%); placebo, 4 out of 10 patients (40\%); and vedolizumab 
Table 4. Treatment-Emergent Adverse Events (SAS-Combined)

\begin{tabular}{|c|c|c|c|c|c|c|c|c|c|c|}
\hline \multirow{3}{*}{ TEAE } & \multirow{2}{*}{\multicolumn{2}{|c|}{$\begin{array}{c}\text { Induction phase } \\
\begin{array}{c}\text { Vedolizumab IV } \\
(n=27)\end{array}\end{array}$}} & \multicolumn{6}{|c|}{ Maintenance phase } & \multirow{2}{*}{\multicolumn{2}{|c|}{$\begin{array}{l}\text { Total } \\
(n=49)\end{array}$}} \\
\hline & & & \multicolumn{2}{|c|}{$\begin{array}{l}\text { Placebo } \\
(n=10)\end{array}$} & \multicolumn{2}{|c|}{$\begin{array}{l}\text { Vedolizumab SC } \\
\qquad(n=10)\end{array}$} & \multicolumn{2}{|c|}{$\begin{array}{l}\text { Vedolizumab IV } \\
\qquad(n=2)\end{array}$} & & \\
\hline & Events & Patients & Events & Patients & Events & Patients & Events & Patients & Events & Patients \\
\hline TEAEs & 37 & $20(74.1)$ & 20 & $10(100.0)$ & 50 & $9(90.0)$ & 9 & $2(100)$ & 116 & 41 (83.7) \\
\hline Related & 1 & $1(3.7)$ & 5 & $4(40.0)$ & 13 & $3(30.0)$ & 1 & $1(50.0)$ & 20 & $9(18.4)$ \\
\hline Not related & 36 & $19(70.4)$ & 15 & $6(60.0)$ & 37 & $6(60.0)$ & 8 & $1(50.0)$ & 96 & 32 (65.3) \\
\hline Mild & 28 & $13(48.1)$ & 17 & $8(80.0)$ & 42 & $5(50.0)$ & 6 & 0 & 93 & $26(53.1)$ \\
\hline Moderate & 8 & $6(22.2)$ & 2 & $1(10.0)$ & 8 & $4(40.0)$ & 3 & $2(100.0)$ & 21 & $13(26.5)$ \\
\hline Severe & 1 & 1 (3.7) & 1 & $1(10.0)$ & 0 & 0 & 0 & 0 & 2 & $2(4.1)$ \\
\hline $\begin{array}{l}\text { Leading to } \\
\text { discontinuation }\end{array}$ & & $2(7.4)$ & & 0 & & 0 & & $1(50.0)$ & & $3(6.1)$ \\
\hline Serious TEAEs & 3 & $3(11.1)$ & 1 & $1(10.0)$ & 1 & $1(10.0)$ & 1 & $1(50.0)$ & 6 & $6(12.2)$ \\
\hline Related & 0 & 0 & 0 & 0 & 0 & 0 & 1 & $1(50.0)$ & 1 & $1(2.0)$ \\
\hline Not related & 3 & $3(11.1)$ & 1 & $1(10.0)$ & 1 & $1(10.0)$ & 0 & 0 & 5 & $5(10.2)$ \\
\hline $\begin{array}{l}\text { Leading to } \\
\text { discontinuation }\end{array}$ & & $2(7.4)$ & & 0 & & 0 & & $1(50.0)$ & & $3(6.1)$ \\
\hline Deaths & & 0 & & 0 & & 0 & & 0 & & 0 \\
\hline
\end{tabular}

Value are presented as number or number $(\%)$.

SAS, safety analysis set; IV, intravenous; SC, subcutaneous; TEAE, treatment-emergent adverse event.

IV, 1 out of 2 patients (50\%) (Table 4). Most AEs were mild to moderate and no deaths occurred. Serious TEAEs were as follows: vedolizumab SC, 1 out of 10 patients (10\%); placebo, 1 out of 10 patients (10\%); and vedolizumab IV, 1 out of 2 patients (50\%). TEAEs leading to discontinuation occurred in 1 out of 2 patients (50\%) in the vedolizumab IV group and in no patients in the other 2 treatment groups. Two patients who received vedolizumab SC experienced an injection-site reaction (20\%) compared with none in those who received placebo or vedolizumab IV (Table 5).

\section{Immunogenicity}

Following vedolizumab IV induction therapy, AVAs were detected in $10 \%(1 / 10)$ of patients who received vedolizumab SC, 30\% (3/10) of patients who received placebo, and in no patients who received vedolizumab IV. None of the AVA-positive patients who received vedolizumab SC were persistently positive or developed neutralizing antibodies. Among AVApositive patients on placebo, $67 \%$ (2/3) were persistently positive and $100 \%$ (3/3) developed neutralizing antibodies. No discernable relationship was observed between AVA status and safety issues that related to injection-site reactions or hypersensitivity reactions (Supplementary Table 3).

\section{DISCUSSION}

The results of this ad-hoc analysis are limited by the small sample size; nevertheless, the results indicate that the efficacy of vedolizumab SC compared with placebo in a subgroup of Japanese patients with UC is consistent with the overall VISIBLE 1 study population. The results for vedolizumab and placebo arms in the subgroup of Japanese patients and overall VISIBLE 1 study population were similar at week 52 for clinical remission, endoscopic improvement, durable clinical response and durable clinical remission, with the exception of corticosteroid-free remission.

The clinical response rate at week 6 (45\%) with vedolizumab IV in the present study was similar to the global VISIBLE population at week $6(56 \%){ }^{17}$ In addition, the higher clinical remission rate at week 52 in patients who were TNF- $\alpha$ antagonist therapy naïve and received vedolizumab SC compared with placebo ( $60 \%$ vs. $25 \%$, respectively) is consistent with that reported at week 60 in Japanese patients who received vedolizumab IV compared with placebo ( $54 \%$ vs. $36 \%$, respectively). ${ }^{11}$

The safety and tolerability of vedolizumab SC in patients with UC was generally favorable with no safety issues that limited treatment, and was consistent with the overall VISIBLE 1 population. ${ }^{17}$ In Japan, the SC administration of other biologics 
Table 5. Most Frequent ( $\geq 5 \%$ ) TEAEs by System Organ Class and Preferred Term (SAS)

\begin{tabular}{|c|c|c|c|c|}
\hline Variable & $\begin{array}{l}\text { Placebo } \\
(n=10)\end{array}$ & $\begin{array}{c}\text { Vedolizumab SC } \\
\qquad(n=10)\end{array}$ & $\begin{array}{c}\text { Vedolizumab IV } \\
(n=2)\end{array}$ & $\begin{array}{c}\text { Total } \\
(n=22)\end{array}$ \\
\hline Patients with any most frequent TEAEs & $10(100.0)$ & $9(90.0)$ & $2(100.0)$ & $21(95.5)$ \\
\hline Blood and lymphatic system disorders & $1(10.0)$ & $1(10.0)$ & $1(50.0)$ & $3(13.6)$ \\
\hline Iron deficiency anemia & $1(10.0)$ & $1(10.0)$ & 0 & $2(9.1)$ \\
\hline Anemia & 0 & 0 & $1(50.0)$ & $1(4.5)$ \\
\hline Eye disorders & 0 & 0 & $2(100.0)$ & $2(9.1)$ \\
\hline Cataract & 0 & 0 & $1(50.0)$ & $1(4.5)$ \\
\hline Conjunctival hyperemia & 0 & 0 & $1(50.0)$ & $1(4.5)$ \\
\hline Gastrointestinal disorders & $4(40.0)$ & $2(20.0)$ & $1(50.0)$ & $7(31.8)$ \\
\hline UC & $2(20.0)$ & 0 & $1(50.0)$ & $3(13.6)$ \\
\hline Abdominal pain & $1(10.0)$ & $1(10.0)$ & 0 & $2(9.1)$ \\
\hline Hemorrhoids & $2(20.0)$ & $1(10.0)$ & 0 & $3(13.6)$ \\
\hline Vomiting & $1(10.0)$ & 0 & 0 & $1(4.5)$ \\
\hline General disorders and administration site conditions & 0 & $3(30.0)$ & $1(50.0)$ & $4(18.2)$ \\
\hline Pyrexia & 0 & 0 & $1(50.0)$ & $1(4.5)$ \\
\hline Injection-site reaction & 0 & $2(20.0)$ & 0 & $2(9.1)$ \\
\hline Peripheral edema & 0 & $1(10.0)$ & 0 & $1(4.5)$ \\
\hline Hepatobiliary disorders & 0 & 0 & $1(50.0)$ & $1(4.5)$ \\
\hline Abnormal hepatic function & 0 & 0 & $1(50.0)$ & $1(4.5)$ \\
\hline Infections and infestations & $4(40.0)$ & $8(80.0)$ & $1(50.0)$ & $13(59.1)$ \\
\hline Adenoviral conjunctivitis & 0 & $1(10.0)$ & 0 & $1(4.5)$ \\
\hline Periodontitis & 0 & $1(10.0)$ & 0 & $1(4.5)$ \\
\hline Oral herpes & 0 & $1(10.0)$ & 0 & $1(4.5)$ \\
\hline Influenza & 0 & $2(20.0)$ & 0 & $2(9.1)$ \\
\hline Pneumonia & 0 & $1(10.0)$ & 0 & $1(4.5)$ \\
\hline Paronychia & 0 & $1(10.0)$ & 0 & $1(4.5)$ \\
\hline Nasopharyngitis & $4(40.0)$ & $4(40.0)$ & $1(50.0)$ & $9(40.9)$ \\
\hline Tonsillitis & 0 & $1(10.0)$ & 0 & $1(4.5)$ \\
\hline Injury, poisoning, and procedural complications & $1(10.0)$ & $3(30.0)$ & 0 & $4(18.2)$ \\
\hline Arthropod sting & 0 & $1(10.0)$ & 0 & $1(4.5)$ \\
\hline Wound & 0 & $1(10.0)$ & 0 & $1(4.5)$ \\
\hline Tooth fracture & 0 & $1(10.0)$ & 0 & $1(4.5)$ \\
\hline Rib fracture & $1(10.0)$ & 0 & 0 & $1(4.5)$ \\
\hline Investigations & 0 & $1(10.0)$ & 0 & $1(4.5)$ \\
\hline Fecal calprotectin increased & 0 & $1(10.0)$ & 0 & $1(4.5)$ \\
\hline Metabolism and nutrition disorders & $1(10.0)$ & 0 & 0 & $1(4.5)$ \\
\hline Alcohol intolerance & $1(10.0)$ & 0 & 0 & $1(4.5)$ \\
\hline Musculoskeletal and connective tissue disorders & $1(10.0)$ & $2(20.0)$ & 0 & $3(13.6)$ \\
\hline Arthralgia & 0 & $1(10.0)$ & 0 & $1(4.5)$ \\
\hline Back pain & $1(10.0)$ & $1(10.0)$ & 0 & $2(9.1)$ \\
\hline Nervous system disorders & $3(30.0)$ & 0 & 0 & $3(13.6)$ \\
\hline Headache & $3(30.0)$ & 0 & 0 & $3(13.6)$ \\
\hline
\end{tabular}


Table 5. Continued

\begin{tabular}{|c|c|c|c|c|}
\hline Variable & $\begin{array}{l}\text { Placebo } \\
(n=10)\end{array}$ & $\begin{array}{c}\text { Vedolizumab SC } \\
\qquad(n=10)\end{array}$ & $\begin{array}{c}\text { Vedolizumab IV } \\
(n=2)\end{array}$ & $\begin{array}{c}\text { Total } \\
(n=22)\end{array}$ \\
\hline Psychiatric disorders & 0 & 1 (10.0) & 0 & $1(4.5)$ \\
\hline Bruxism & 0 & $1(10.0)$ & 0 & $1(4.5)$ \\
\hline Renal and urinary disorders & $1(10.0)$ & 0 & 0 & $1(4.5)$ \\
\hline Proteinuria & $1(10.0)$ & 0 & 0 & $1(4.5)$ \\
\hline Respiratory, thoracic, and mediastinal disorders & $1(10.0)$ & $3(30.0)$ & 0 & $4(18.2)$ \\
\hline Cough & $1(10.0)$ & 0 & 0 & $1(4.5)$ \\
\hline Epistaxis & 0 & $1(10.0)$ & 0 & $1(4.5)$ \\
\hline Sneezing & 0 & $1(10.0)$ & 0 & $1(4.5)$ \\
\hline Upper respiratory tract inflammation & 0 & $1(10.0)$ & 0 & $1(4.5)$ \\
\hline Skin and SC tissue disorders & $1(10.0)$ & $5(50.0)$ & $1(50.0)$ & 7 (31.8) \\
\hline Miliaria & $1(10.0)$ & 0 & 0 & $1(4.5)$ \\
\hline Allergic dermatitis & 0 & $1(10.0)$ & 0 & $1(4.5)$ \\
\hline Atopic dermatitis & 0 & 0 & $1(50.0)$ & $1(4.5)$ \\
\hline Eczema & 0 & $1(10.0)$ & 0 & $1(4.5)$ \\
\hline Asteatotic eczema & 0 & $1(10.0)$ & 0 & $1(4.5)$ \\
\hline Prurigo & 0 & $1(10.0)$ & 0 & $1(4.5)$ \\
\hline Seborrheic dermatitis & 0 & $1(10.0)$ & 0 & $1(4.5)$ \\
\hline Rash pruritic & 0 & $1(10.0)$ & 0 & $1(4.5)$ \\
\hline Pustular psoriasis & 0 & 0 & $1(50.0)$ & $1(4.5)$ \\
\hline Vascular disorders & 0 & 1 (10.0) & 0 & $1(4.5)$ \\
\hline Flushing & 0 & $1(10.0)$ & 0 & $1(4.5)$ \\
\hline
\end{tabular}

Value are presented as number (\%).

TEAE, treatment-emergent adverse event; SAS, safety analysis set; SC, subcutaneous; IV, intravenous; UC, ulcerative colitis.

in patients with UC or CD has also been reported as safe, with similar safety profiles to their overall study populations. ${ }^{1,19} \mathrm{In}$ jection-site reactions occurred in 2 patients (20\%) who received vedolizumab SC and in no patients who received vedolizumab IV or placebo. In the present analysis, the rate of injectionsite reactions observed in patients who received vedolizumab SC was broadly in line with that in the overall VISIBLE 1 study population ${ }^{17}$ and with other SC treatments for patients with IBD (ranging from $3 \%$ to $20 \%$ ). ${ }^{20-22}$

The proportion of patients who received vedolizumab SC and were positive for AVA was consistent with that reported for the overall VISIBLE 1 population ${ }^{17}$ and the pooled GEMINI 1 and GEMINI 2 population. ${ }^{23}$ The proportion of patients who received vedolizumab SC and were positive for AVA (10\%) was within the range of anti-drug antibody formation rates $(0-65 \%)$ observed with other biologics in patients with IBD. ${ }^{24}$ No relationship was observed between AVA status and infusion-related reactions and hypersensitivity reactions.
PROs improved following vedolizumab IV during induction therapy. While improvements in PROs were maintained throughout the maintenance treatment phase with vedolizumab SC and IV, they were not maintained with placebo. The improvements in PROs were consistent with results for efficacy, safety, and tolerability endpoints, and may benefit patients' perceived quality of life. The improvements in PROs in Japanese patients who received vedolizumab SC were also consistent with those in health-related quality of life measures in the overall VISIBLE 1 study population who received vedolizumab SC, ${ }^{17}$ those in patients with UC in GEMINI 1 who received vedolizumab $\mathrm{IV}^{25}$ and those in Japanese patients with other biologics. ${ }^{26,27}$

The results of the present analysis indicate that vedolizumab SC may provide a similar level of clinical benefit to that with vedolizumab IV in terms of long-term efficacy and safety, and indicate that both routes of administration are also viable options for Japanese patients. Recently, the phase $3 \mathrm{~b}$ random- 
ized, double-blind VARSITY study evaluated the efficacy and safety of vedolizumab IV compared with adalimumab SC at week 52 in patients with moderate to severe UC. ${ }^{28}$ The primary endpoint was met with a significantly higher proportion of patients with clinical remission (complete Mayo score $\leq 2$ with no subscore $>1$ ) at week 52 in the vedolizumab group (31\%) than the adalimumab group $(23 \% ; P=0.0061){ }^{28}$ Headto-head studies of vedolizumab SC with other biologics administered via the SC route are required.

A nationwide web-based survey on preferences for shared decision making (SDM) was conducted in Japanese patients with IBD. ${ }^{29}$ Results showed that patients receiving biologics significantly favored SDM compared with those not receiving biologics, that patients with history of surgery significantly favored SDM compared with those without, and that most patients treated at university hospitals favored SDM. ${ }^{29}$ In addition, a clear correlation was observed between the level of agreement that patients and their doctors had regarding SDM and patients' overall satisfaction with their treatment. ${ }^{30}$ Having both SC and IV routes of administration as available treatment options may help physicians to tailor treatment decisions and thus maximize patient compliance and improve clinical outcomes. Further surveys on routes of administration, patient preferences, and outcomes are needed to inform physicians in Japan.

Studies both in and outside of Japan have evaluated patient satisfaction and patient adherence with regard to biologics. A multicenter questionnaire survey was conducted in Japanese patients with CD to assess their satisfaction with and adherence to self-injections of adalimumab. ${ }^{31}$ Results showed that $38 \%$ of patients would not accept self-injection therapy before treatment initiation; however, $75 \%$ of patients were satisfied with the treatment after treatment initiation. ${ }^{31}$ In addition, patient adherence to self-injection therapy with adalimumab was high at $85 \%{ }^{31}$ A hospital claims database analysis in Japanese patients with rheumatoid arthritis indicated that while the administration route does not have a significant association with the initiation of biologics, it does play a major role in switches between biologic agents. ${ }^{32}$ A post-marketing surveillance study of self-injection of methotrexate SC in German patients with rheumatoid arthritis or psoriatic arthritis found that self-injection led to patients feeling more independence (89\%) and a better quality of life $(84 \%) .{ }^{33}$ Finally, a retrospective, longitudinal analysis of a cohort of patients with UC in the Japan Medical Data Center claims database evaluated the long-term persistence associated with adalimumab or infliximab combined with an immunomodulator (adminis- tered as step-up treatment or simultaneously) compared with adalimumab or infliximab alone during maintenance therapy. Results showed that in patients with UC who had been newly prescribed either infliximab IV or adalimumab SC, the proportion who switched treatment was similar at $\sim 10 \% .{ }^{34}$ However, this study did not investigate the motivational aspects of the switch. ${ }^{34}$ These studies, including those from other disease areas, suggest that SC administration could be a useful option to meet various individual preferences and potentially improve patient satisfaction.

A limitation of this post hoc analysis is the small number of Japanese patients enrolled in the VISIBLE 1 study, which prevented meaningful analyses of pharmacokinetics and of the vedolizumab IV subgroup. This limitation was compounded by the overall sample size of VISIBLE 1, which was smaller than GEMINI 1 study of vedolizumab IV in patients with UC. ${ }^{13}$ In addition, this limitation may have contributed to the lack of statistically significant differences (despite numerically greater differences) between treatment groups for some efficacy endpoints, as well as the numerically lower proportion of patients with corticosteroid-free clinical remission in the vedolizumab SC group than in the placebo group. Analysis in a larger number of Japanese patients would also enable the efficacy of vedolizumab in those with prior TNF- $\alpha$ antagonist use to be determined. Another limitation is that outcomes of SC maintenance were examined in week 6 responders, after only 2 induction infusions. Therefore, this overall study may have selected patients who responded very well to vedolizumab, and in turn influenced the results of the present analysis. Further study is also likely to be needed to determine the rate of injection-site reactions with vedolizumab SC.

In conclusion, the new vedolizumab SC formulation (108 mg Q2W) exhibited similar trends in efficacy as maintenance therapy in Japanese patients with UC compared with the overall VISIBLE 1 study population, as did vedolizumab IV as induction therapy. ${ }^{17}$ The safety and tolerability of vedolizumab SC were generally similar to the whole study population. ${ }^{17}$ Further study of vedolizumab SC in a prospective, specifically designed study of a larger number of Japanese patients with UC is warranted, as well as in patients with UC who are not Japanese or Caucasian.

\section{ADDITIONAL INFORMATION}

\section{Funding Source}

This research funded by Takeda Pharmaceutical Co. Ltd. 


\section{Conflict of Interest}

Hibi T has received payment for lectures including service on speakers bureaus from Aspen Japan, AbbVie, Ferring, Gilead Sciences, Janssen, JIMRO, Kissei Pharmaceutical, Mitsubishi Tanabe Pharma, Mochida Pharmaceutical, Nippon Kayaku, Pfizer, Takeda Pharmaceutical, and Zeria Pharmaceutical; and has received grants from AbbVie, EA Pharma, JIMRO, Otsuka Holdings, and Zeria Pharmaceutical. Kobayashi T has received personal fees as an advisor from Alfresa Pharma, Covidien, Eli Lilly, Ferring Pharmaceuticals, Janssen, Kyorin Pharmaceutical, Mochida Pharmaceutical, Nippon Kyaku, Pfizer, Takeda Pharmaceutical, Thermo Scientific, AbbVie, Gilead Sciences, Celltrion, and EA Pharma; personal fees for lectures from AbbVie, Pfizer, Astellas, Alfresa Pharma, Cellitron, EA Pharma, Kyorin Pharmaceutical, Nippon Kyaku, Mochida Pharmaceutical, Takeda Pharmaceutical, Mitsubishi Tanabe Pharma, Zeria Pharmaceutical, Gilead Sciences, Janssen, JIMRO, and Covidien; and grants from JIMRO, AbbVie, EA Pharma, Zeria Pharmaceutical, Kyorin Pharmaceutical, Mochida Pharmaceutical, Thermo Fisher Scientific, Alfresa Pharma, and Nippon Kayaku. Ito H has received payment for lectures including service on speakers bureaus from Mitsubishi Tanabe Pharma, AbbVie, Takeda Pharmaceutical, EA Pharma, Zeria Pharmaceutical, Janssen, Mochida Pharmaceutical, Nippon Kayaku, and Pfizer. Ashida T has received payment for lectures including service on speakers bureaus from Takeda Pharmaceutical, Mitsubishi Tanabe Pharma, and Kissei Pharmaceutical. Yokoyama $\mathrm{T}$ has received grants from Mochida Pharmaceutical, Takeda Pharmaceutical, AbbVie, EA Pharma, and Ferring Pharmaceuticals. Nagahori M has received consultancy fees from Takeda Pharmaceutical; and payment for lectures including service on speakers bureaus from Kissei Pharmaceutical, and Takeda Pharmaceutical, Kyorin Pharmaceutical, Mochida Pharmaceutical, AbbVie, Mitsubishi Tanabe Pharma, Nippon Kayaku, Asahi Kasei Medical, Zeria Pharmaceutical, Astellas, Nichi-Iko, and Janssen. Watanabe M has received payment for lectures including service on speakers bureaus from Mitsubishi Tanabe Pharma, Takeda Pharmaceutical, EA Pharma, Zeria Pharmaceutical, Janssen, Gilead Sciences, Celltrion Healthcare, and Pfizer; and grants from EA Pharma, Zeria Pharmaceutical, Mitsubishi Tanabe Pharma, Takeda Pharmaceutical, Nippon Kayaku, Mochida, Kissei, Miyarisan, Asahi Kasei, JIMRO, Kyorin Pharmaceutical, AbbVie, Kyowa Hakko Kirin, Alfresa, Ayumi, Astellas, MSD, Daiichi Sankyo, Taiho, Toray, Chugai, Gilead Sciences, and Fujirebio. Shikamura M, Yamaguchi T, Hori T, and Pinton P are employed by Takeda
Pharmaceutical. Inaba T has nothing to declare.

Hibi $\mathrm{T}$ and Watanabe $\mathrm{M}$ are editorial board members of the journal but were not involved in the peer reviewer selection, evaluation, or decision process of this article. No other potential conflicts of interest relevant to this article were reported.

\section{Author Contribution}

Conception and design, data analysis and interpretation, and manuscript drafting and writing: Kobayashi T, Shikamura M, Yamaguchi T, Hori T, Pinton P. Data acquisition and manuscript writing: Ito H, Ashida T, Yokoyama T, Nagahori M, Inaba T, Watanabe M, Hibi T. All authors had access to the study data, reviewed and approved the final version of this manuscript, and agree to be accountable for all aspects of the work, which includes ensuring that questions related to the accuracy or integrity of any part of the work are appropriately investigated and resolved.

\section{Others}

The authors are grateful to the patients who participated in the trial and their families, investigators and the staff at the following study sites in Japan who supported this study: Ehime Prefectural Central Hospital; Fukuoka University Chikushi Hospital; Fukuoka University Hospital; Hamamatsu Minami Hospital; Hiroshima University Hospital; Infusion Clinic; Kagawa Prefectural Central Hospital; Keio University Hospital; Kitasato University Kitasato Institute Hospital; Kurume University Hospital; Kyorin University Hospital; Ofuna Chuo Hospital; Okayama University Hospital; Osaka City General Hospital; Saga University Hospital; Sapporo Higashi Tokushukai Hospital; Sapporo Kosei General Hospital; Sapporo Tokushukai Hospital; Shiga University of Medical Science Hospital; The Jikei University Hospital; Toho University Medical Center, Sakura Hospital; Tokyo Medical And Dental University; Tokyo Yamate Medical Center; Wakayama Medical University Hospital and Yokoyama IBD Clinic. The authors acknowledge the contribution of Eri Udagawa to development of this manuscript. Medical writing support was provided by Nicholas Crabb, MSc, of FireKite, an Ashfield company, part of UDG Healthcare plc, during the development of this manuscript, which was funded by Takeda Pharmaceutical Company Limited in compliance with Good Publication Practice 3 ethical guidelines (Battisti WP, et al. Ann Intern Med 2015;163:461-464).

\section{ORCID}

Kobayashi T https://orcid.org/0000-0002-2073-4234 
Ito $\mathrm{H}$

Ashida T

Yokoyama T

Nagahori M

Inaba $\mathrm{T}$

Shikamura M

Yamaguchi T

Hori T

Pinton P

Watanabe M

Hibi T
https://orcid.org/0000-0001-8503-141X https://orcid.org/0000-0003-4553-7664

https://orcid.org/0000-0003-3689-1995 https://orcid.org/0000-0002-5323-7058 https://orcid.org/0000-0003-0936-5834 https://orcid.org/0000-0002-8980-5811 https://orcid.org/0000-0003-3045-7215 https://orcid.org/0000-0003-0638-6664 https://orcid.org/0000-0002-4227-7687 https://orcid.org/0000-0002-5475-9544 https://orcid.org/0000-0002-6256-1204

\section{Supplementary Material}

Supplementary materials are available at the Intestinal Research website (https://www.irjournal.org).

\section{REFERENCES}

1. Hibi T, Imai Y, Senoo A, Ohta K, Ukyo Y. Efficacy and safety of golimumab 52-week maintenance therapy in Japanese patients with moderate to severely active ulcerative colitis: a phase 3, double-blind, randomized, placebo-controlled study (PURSUIT-J study). J Gastroenterol 2017;52:1101-1111.

2. Testa A, Castiglione F, Nardone OM, Colombo GL. Adherence in ulcerative colitis: an overview. Patient Prefer Adherence 2017;11:297-303.

3. Bittner B, Richter W, Schmidt J. Subcutaneous administration of biotherapeutics: an overview of current challenges and opportunities. BioDrugs 2018;32:425-440.

4. Pharmaceuticals and Medical Devices Agency. Vedolizumab prescribing information [Internet]. c2019 [cited 2020 May 26]. https://www.info.pmda.go.jp/go/pack/2399405F1020_1_03/.

5. Pharmaceuticals and Medical Devices Agency. Infliximab prescribing information [Internet]. c2019 [cited 2020 May 26]. https://www.info.pmda.go.jp/go/pack/2399402F1026_1_43/.

6. Pharmaceuticals and Medical Devices Agency. Adalimumab prescribing information [Internet]. c2020 [cited 2020 May 26]. https://www.info.pmda.go.jp/go/pack/3999459G1029_1_02/.

7. Pharmaceuticals and Medical Devices Agency. Golimumab prescribing information [Internet]. c2020 [cited 2020 May 26]. https://www.info.pmda.go.jp/go/pack/3999433G1024_1_21/.

8. Pharmaceuticals and Medical Devices Agency. Ustekinumab prescribing information [Internet]. c2020 [cited 2020 May 26]. https://www.info.pmda.go.jp/go/pack/3999431G1025_1_17/.

9. Soler D, Chapman T, Yang LL, Wyant T, Egan R, Fedyk ER. The binding specificity and selective antagonism of vedolizumab,

an anti-alpha4beta7 integrin therapeutic antibody in development for inflammatory bowel diseases. J Pharmacol Exp Ther 2009;330:864-875.

10. Wyant T, Fedyk E, Abhyankar B. An overview of the mechanism of action of the monoclonal antibody vedolizumab. J Crohns Colitis 2016;10:1437-1444.

11. Motoya S, Watanabe K, Ogata H, et al. Vedolizumab in Japanese patients with ulcerative colitis: a phase 3, randomized, double-blind, placebo-controlled study. PLoS One 2019;14: e0212989.

12. Watanabe K, Motoya S, Ogata H, et al. Effects of vedolizumab in Japanese patients with Crohn's disease: a prospective, multicenter, randomized, placebo-controlled phase 3 trial with exploratory analyses. J Gastroenterol 2020;55:291-306.

13. Feagan BG, Rutgeerts P, Sands BE, et al. Vedolizumab as induction and maintenance therapy for ulcerative colitis. N Engl J Med 2013;369:699-710.

14. Kobayashi T, Uda A, Udagawa E, Hibi T. Lack of increased risk of lymphoma by thiopurines or biologics in Japanese patients with inflammatory bowel disease: a large-scale administrative database analysis. J Crohns Colitis 2020;14:617-623.

15. Beaugerie L, Brousse N, Bouvier AM, et al. Lymphoproliferative disorders in patients receiving thiopurines for inflammatory bowel disease: a prospective observational cohort study. Lancet 2009;374:1617-1625.

16. Pasternak B, Svanström H, Schmiegelow K, Jess T, Hviid A. Use of azathioprine and the risk of cancer in inflammatory bowel disease. Am J Epidemiol 2013;177:1296-1305.

17. Sandborn WJ, Baert F, Danese S, et al. Efficacy and safety of vedolizumab subcutaneous formulation in a randomized trial of patients with ulcerative colitis. Gastroenterology 2020;158: 562-572.

18. Rosario M, Yang LL, Wyant T. P010 Results from a new antivedolizumab antibody assay. Am J Gastroenterol 2019;114:S3.

19. Hibi T, Imai Y, Murata Y, Matsushima N, Zheng R, Gasink C. Efficacy and safety of ustekinumab in Japanese patients with moderately to severely active Crohn's disease: a subpopulation analysis of phase 3 induction and maintenance studies. Intest Res 2017;15:475-486.

20. AbbVie Inc. HUMIRA ${ }^{\circledast}$ (Adalimumab) prescribing information. North Chicago: AbbVie Inc., 2018.

21. UCB Inc. CIMZIA* (certolizumab pegol) prescribing information. Smyrna: UCB Inc., 2018.

22. Janssen Biotech Inc. SIMPONI ${ }^{\circledR}$ (golimumab) prescribing information. Horsham: Janssen Biotech Inc., 2018.

23. Colombel JF, Sands BE, Rutgeerts P, et al. The safety of vedoli- 
zumab for ulcerative colitis and Crohn's disease. Gut 2017;66: 839-851.

24. Vermeire S, Gils A, Accossato P, Lula S, Marren A. Immunogenicity of biologics in inflammatory bowel disease. Therap Adv Gastroenterol 2018;11:1756283X17750355.

25. Feagan BG, Patel H, Colombel JF, et al. Effects of vedolizumab on health-related quality of life in patients with ulcerative colitis: results from the randomised GEMINI 1 trial. Aliment Pharmacol Ther 2017;45:264-275.

26. Hibi T, Motoya S, Ashida T, et al. Efficacy and safety of abrilumab, an $\alpha 4 \beta 7$ integrin inhibitor, in Japanese patients with moderate-to-severe ulcerative colitis: a phase II study. Intest Res 2019;17:375-386.

27. Suzuki Y, Motoya S, Hanai H, et al. Efficacy and safety of adalimumab in Japanese patients with moderately to severely active ulcerative colitis. J Gastroenterol 2014;49:283-294.

28. Sands BE, Peyrin-Biroulet L, Loftus EV, et al. 416a -Vedolizumab shows superior efficacy versus adalimumab: results of varsity-the first head-to-head study of biologic therapy for moderate-to-severe ulcerative colitis. Gastroenterol 2019;156: S-81.

29. Morishige R, Nakajima H, Yoshizawa K, Mahlich J, Sruamsiri
R. Preferences regarding shared decision-making in Japanese inflammatory bowel disease patients. Adv Ther 2017;33:22422256.

30. Mahlich J, Matsuoka K, Sruamsiri R. Shared decision making and treatment satisfaction in Japanese patients with inflammatory bowel disease. Dig Dis 2017;35:454-462.

31. Hirai F, Watanabe K, Matsumoto T, et al. Patients' assessment of adalimumab self-injection for Crohn's disease: a multicenter questionnaire survey (The PEARL Survey). Hepatogastroenterology 2014;61:1654-1660.

32. Mahlich J, Sruamsiri R. Treatment patterns of rheumatoid arthritis in Japanese hospitals and predictors of the initiation of biologic agents. Curr Med Res Opin 2017;33:101-107.

33. Striesow F, Brandt A. Preference, satisfaction and usability of subcutaneously administered methotrexate for rheumatoid arthritis or psoriatic arthritis: results of a postmarketing surveillance study with a high-concentration formulation. Ther Adv Musculoskelet Dis 2012;4:3-9.

34. Kobayashi T, Udagawa E, Uda A, Hibi T, Hisamatsu T. Impact of immunomodulator use on treatment persistence in patients with ulcerative colitis: a claims database analysis. J Gastroenterol Hepatol 2020;35:225-232. 
See "Efficacy and safety of a new vedolizumab subcutaneous formulation in Japanese patients with moderately to severely active ulcerative colitis" on page 448-460.

\section{SUPPLEMENTARY MATERIAL}

\section{Subcutaneous Administration of Vedolizumab and Placebo}

After receiving training from the health care provider (HCP; investigator or designee) on the proper SC injection technique and management of hypersensitivity reactions which may occur with the injection, patients or their caregivers injected vedolizumab subcutaneous (SC) or placebo SC under HCP supervision at clinic visits on Weeks 6 and 8. Blinding was maintained in the maintenance phase, and only the pharmacists at the study site were aware of treatment assignments.

During visits on Week 6 and 8, HCPs ensured proper injection technique prior to home dosing and observed for any potential hypersensitivity or injection site reactions associated with SC injection. Patients and caregivers could attend the clinic at Weeks 10 and 12 if further training was required. Patients or their caregivers also administered SC injections during the scheduled clinic visits at Weeks 14, 22, 30, 38, 46, and 50 , under the supervision of the HCP, to allow continued observation of injection technique and adverse events (AEs); while all other scheduled SC injections occurred outside of the clinic. All intravenous (IV) infusions were administered by a HCP during clinic visits at Weeks 6, 14, 22, 30, 38, and 46. HCPs had appropriate monitoring and treatment for hypersensitivity reactions available for use following administration of study drug. Patients who experienced a severe hypersensitivity reaction associated with study drug administration were discontinued from the study.

Patients and their caregivers were instructed to inject SC doses into the thigh, abdomen, or upper arm, and to rotate the injection sites. Patients and their caregivers were instructed that the upper arm was to be used only when the caregiver administers the SC injection. For all SC dosing occurring outside of the clinic, patients received a phone call from their HCP within 24 hours prior to every injection to administer the progressive multifocal leukoencephalopathy (PML) subjective checklist and enquire about general health status and experience with prior injections. In accordance with the Risk Assessment and Management Plan for PML (RAMP), any posi- tive PML subjective finding was evaluated via the physician administered PML objective checklist prior to the subject receiving the respective dose (refer to RAMP Site Staff Brochure). Patients also received a phone call from their HCP within 12 hours after the home injection at Weeks 10 and 12 to enquire about health status and experience with injection unless they attended the clinic on these days.

\section{Corticosteroid Tapering Regimen}

For prednisone at doses $>10 \mathrm{mg} /$ day (or equivalent), the dose was reduced at a rate of $5 \mathrm{mg} /$ week until a $10 \mathrm{mg}$ /day dose was reached. For prednisone at doses $\leq 10 \mathrm{mg} /$ day (or equivalent) or a $10 \mathrm{mg} /$ day dose (or equivalent) was achieved by tapering, the dose was reduced at a rate of $2.5 \mathrm{mg} /$ week until discontinuation. For patients who could not tolerate the corticosteroid taper without recurrence of clinical symptoms, corticosteroids were increased up to the original dose at the start of induction therapy (without exceeding the baseline dose). In such cases, the tapering regimen was reinitiated within 2 weeks. Patients whose corticosteroid concentration consistently could not be tapered were withdrawn from the study.

\section{Assessments}

For disease activity assessment, baseline complete Mayo scores were obtained within 10 days prior to enrollment, using subject diary entries within the 10 days prior to enrollment and flexible sigmoidoscopy results obtained during the screening period. Sigmoidoscopy was conducted at Weeks 6 and 52 (or end-of-treatment visit), and a complete Mayo score was calculated for these visits for endpoint assessments. All endoscopies (Week 0, 6, and 52) were assessed centrally. Complete Mayo scores at Week 0,6 , and 52 were calculated by the investigator or designee, and the endoscopic component subscore was provided by the central reader.

\section{Patient-Reported Outcomes}

The Inflammatory Bowel Disease Questionnaire (IBDQ) is comprised of 32 questions that cover 4 domains of health-related quality of life, as follows: bowel symptoms (10 items), emotional function (12 items), social function (5 items), and 
systemic function (5 items). Each question is rated on a scale of 1 to 7 . A total IBDQ score is calculated by adding the scores from each domain (total IBDQ score ranging from 32 to 224). The EuroQol-5 Dimension visual analog scale score is a selfassessment questionnaire of overall health that uses a $20 \mathrm{~cm}$ visual, vertical scale, with a score ranging from 0 (worst) to 100 (best possible) health. The Work Productivity and Activity Impairment-Ulcerative Colitis (WPAI-UC) instrument consists of 4 metrics, as follows: absenteeism (percentage of work time missed owing to health-related issues in the past 7 days), presenteeism (percentage of impairment experienced owing to health-related issues while at work in the past 7 days), overall work productivity loss (an overall impairment estimate that is comprised of absenteeism and presenteeism), and activity impairment (percentage of impairment in daily activities owing to health-related issues in the past 7 days). Higher WPAIUC percentages indicate greater impairment and lower productivity (i.e., worse outcomes). 
A

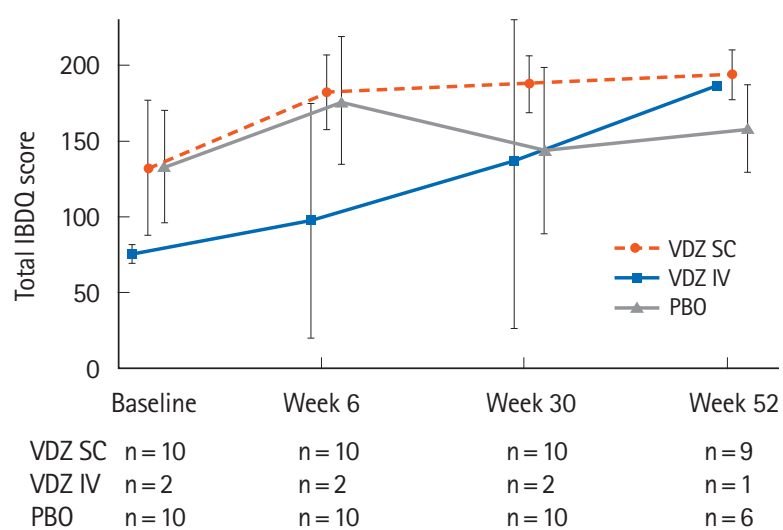

C

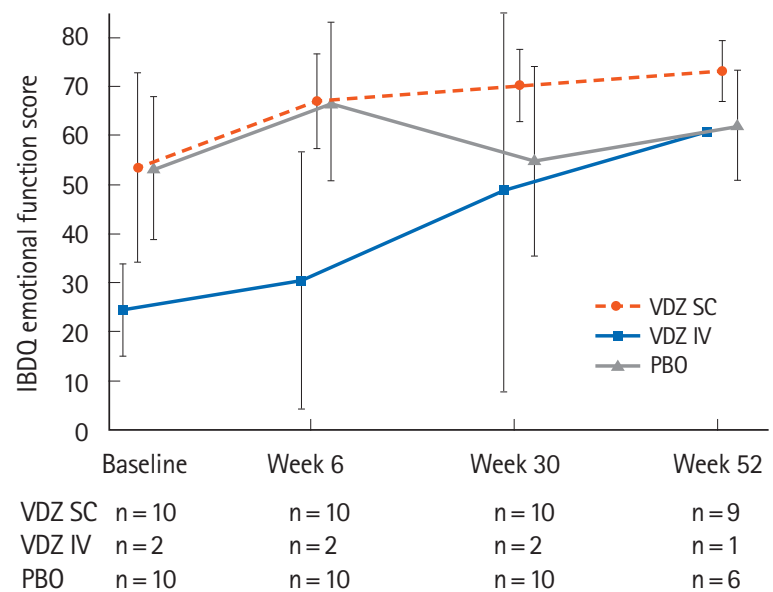

$\boldsymbol{E}$

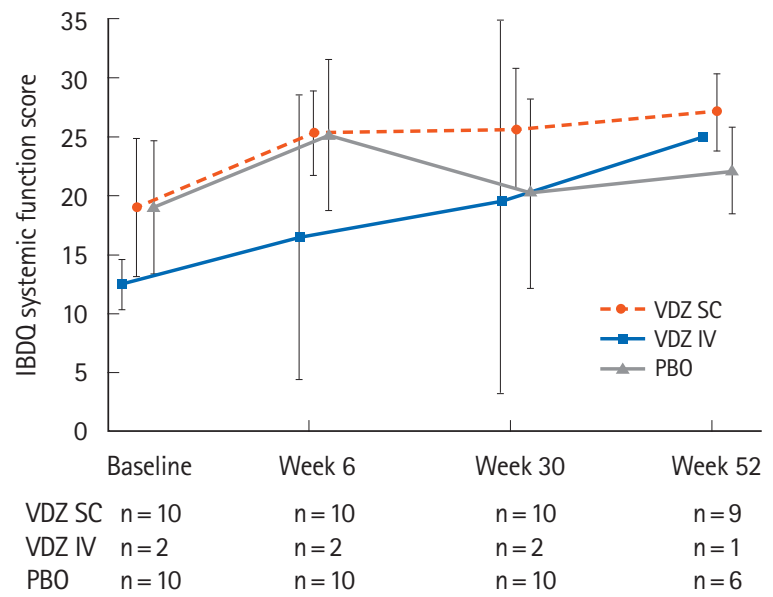

B

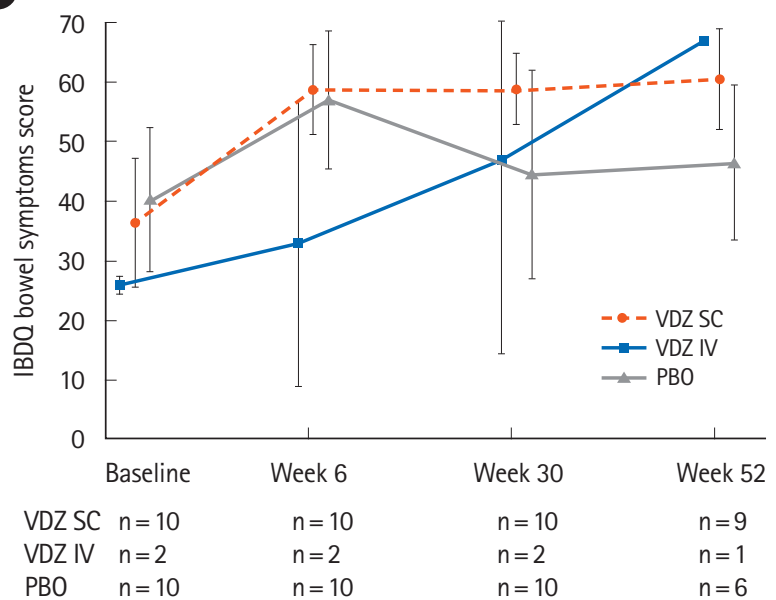

(D)

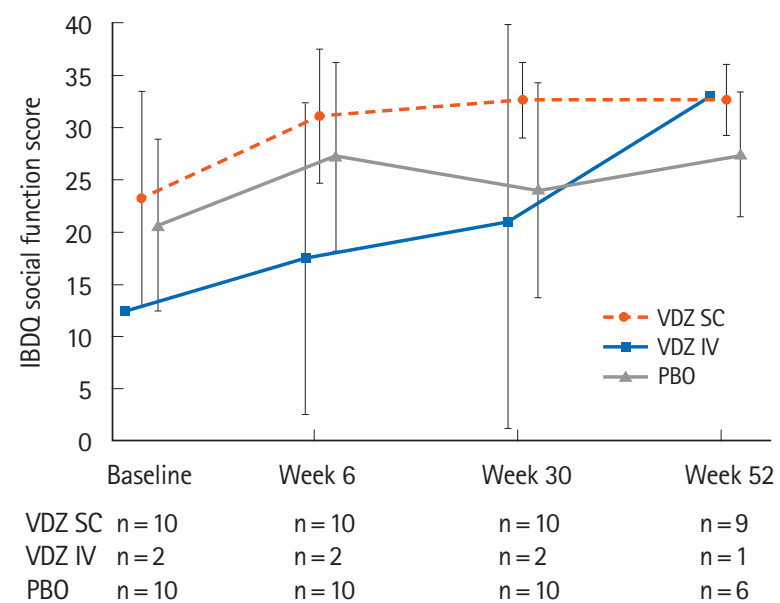

Supplementary Fig. 1. IBDQ total scores by study visit. (A) IBDO total score. (B) IBDO bowel symptoms domain score. (C) IBDO emotional function domain score. (D) IBDQ social function domain score. (E) IBDQ systemic symptoms score. Data are from the full analysis set, with last observation carried forward. The full analysis set comprised of all randomized patients who received $\geq 1$ dose of study drug. The full analysis set did not include patients who only received vedolizumab IV induction treatment and were not randomized into the maintenance phase. Data represent mean and standard deviation. IBDQ, Inflammatory Bowel Disease Questionnaire; VDZ, vedolizumab; SC, subcutaneous; IV, intravenous; PBO, placebo. 


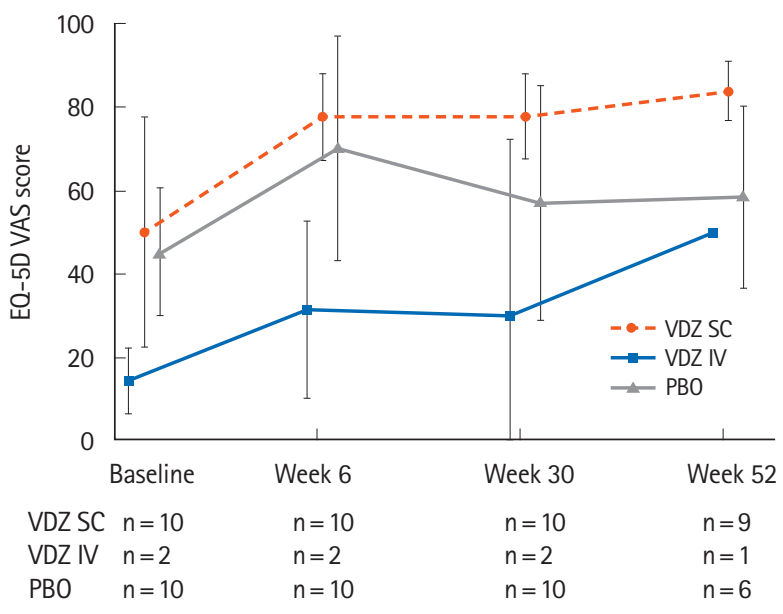

Supplementary Fig. 2. Euro0ol-5 dimension visual analog scale (EQ-5D VAS) score. Data are from the full analysis set, with last observation carried forward. The full analysis set comprised of all randomized patients who received $\geq 1$ dose of study drug. The full analysis set did not include patients who only received vedolizumab IV induction treatment and were not randomized into the maintenance phase. Data represent mean and standard deviation. VDZ, vedolizumab; SC, subcutaneous; IV, intravenous; PBO, placebo.
A

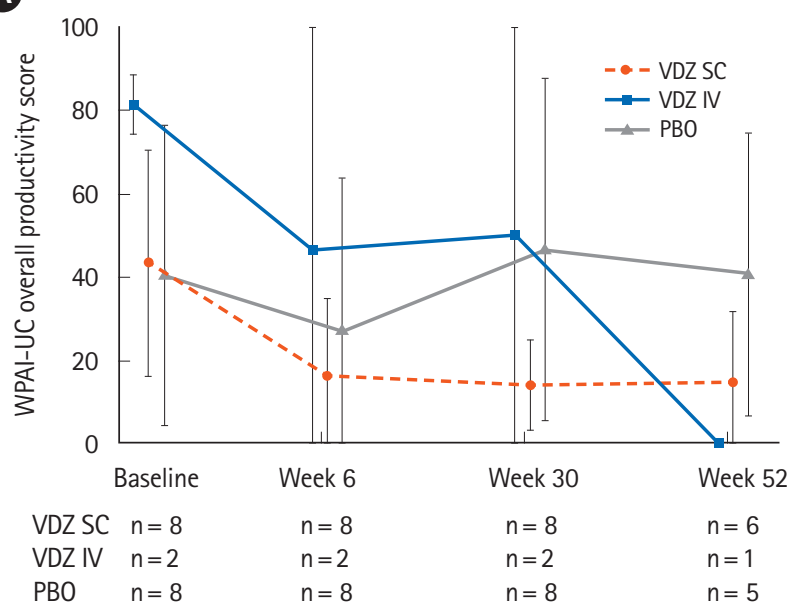

B

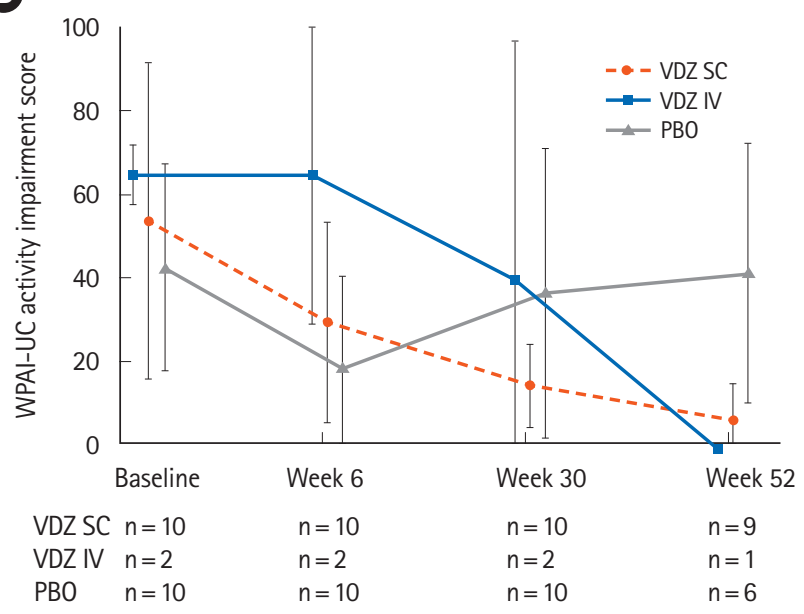

Supplementary Fig. 3. WPAI-UC scores by study visit. (A) WPAIUC overall work productivity score. (B) WPAI-UC activity impairment score. Data are from the full analysis set, with last observation carried forward. The full analysis set comprised of all randomized patients who received $\geq 1$ dose of study drug. The full analysis set did not include patients who only received vedolizumab IV induction treatment and were not randomized into the maintenance phase. Data represent mean and standard deviation. WPAI-UC, Work Productivity and Activity Impairment-ulcerative colitis; VDZ, vedolizumab; SC, subcutaneous; IV, intravenous; PBO, placebo. 
Supplementary Table 1. Demographic and Baseline Characteristics of Patients Enrolled in the VISIBLE 1 Study (SAF-C)

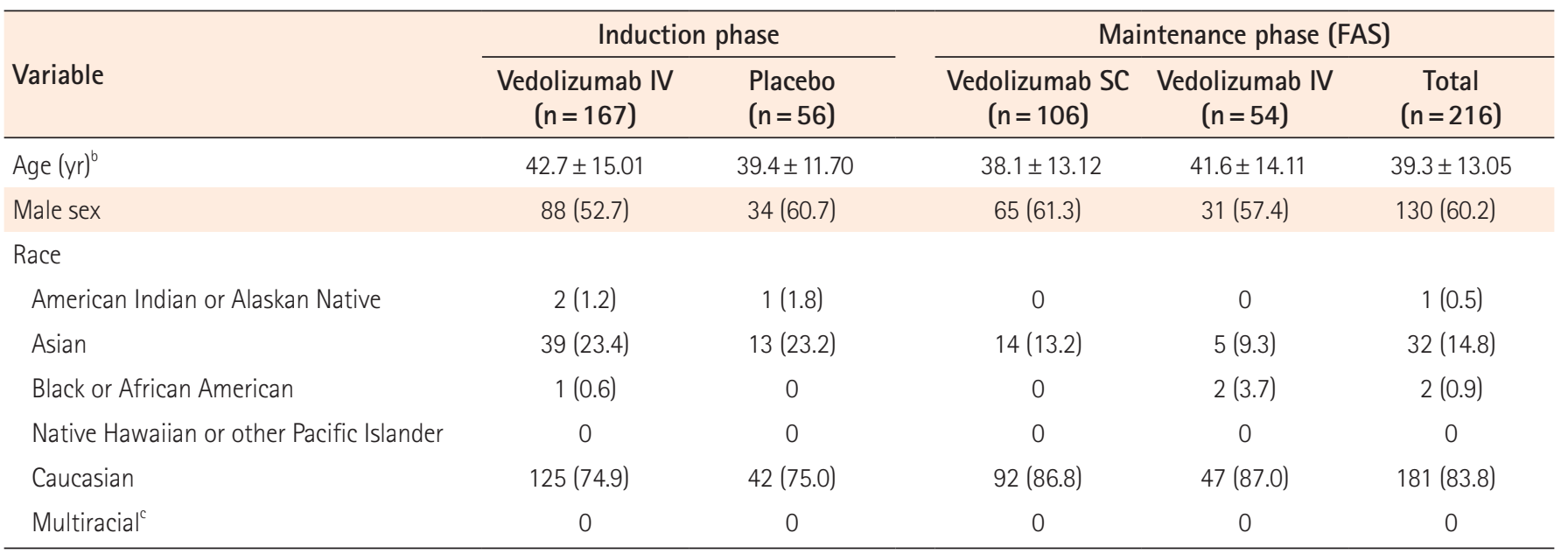

Values are presented as mean \pm standard deviation or number (\%).

${ }^{a}$ Included all randomized patients $(n=216)$ who received at least 1 dose of study drug.

${ }^{6}$ Age at date of informed consent.

'Patient checked more than 1 race option on the case report form.

SAF-C, safety analysis set-combined; FAS, full analysis set; IV, intravenous; SC, subcutaneous.

Supplementary Table 2. Fecal Calprotectin by Visit (FAS)

\begin{tabular}{|c|c|c|c|}
\hline \multirow{2}{*}{ Variable } & \multicolumn{3}{|c|}{ Maintenance phase (FAS) } \\
\hline & Placebo & Vedolizumab SC & Vedolizumab IV \\
\hline Baseline, No. & 10 & 10 & 2 \\
\hline Week 6, No. & 10 & 10 & 2 \\
\hline Median (min, max) $(\mu \mathrm{g} / \mathrm{g})$ & $1,353.5(43,43,503)$ & $374.5(33,76,800)$ & $1,686.5(784,2,589)$ \\
\hline Median $(\min , \max )(\mu \mathrm{g} / \mathrm{g})$ & $322.0(18,73,172)$ & $184.5(15,76,800)$ & 26 \\
\hline Week 52, No. & 2 & 8 & 1 \\
\hline Median (min, max) ( $\mu \mathrm{g} / \mathrm{g})$ & $49.0(48,50)$ & $143.5(32,319)$ & 16 \\
\hline
\end{tabular}

FAS, full analysis set; SC, subcutaneous; IV, intravenous; min, minimum; max maximum. 
Supplementary Table 3. Summary of AVA Status by Infusion-Related Reactions and Hypersensitivity reactions

\begin{tabular}{|c|c|c|c|c|}
\hline \multirow{2}{*}{$\begin{array}{l}\text { Outcome, yes/no } \\
\text { Overall AVA status }\end{array}$} & \multicolumn{2}{|c|}{ Induction phase } & \multicolumn{2}{|c|}{ Maintenance phase } \\
\hline & $\begin{array}{l}\text { Vedolizumab IV } \\
\qquad(n=27)\end{array}$ & $\begin{array}{l}\text { Placebo } \\
(n=10)\end{array}$ & $\begin{array}{c}\text { Vedolizumab SC } \\
\qquad(n=10)\end{array}$ & $\begin{array}{c}\text { Vedolizumab IV } \\
\quad(n=2)\end{array}$ \\
\hline \multicolumn{5}{|l|}{ Yes } \\
\hline No. & 0 & 0 & 0 & 0 \\
\hline AVA positive & 0 & 0 & 0 & 0 \\
\hline \multicolumn{5}{|l|}{ No } \\
\hline No. & 27 & 10 & 10 & 2 \\
\hline AVA negative & 25 (92.6) & $7(70)$ & $9(90)$ & $2(100)$ \\
\hline No. & 2 & 0 & 6 & 1 \\
\hline AVA negative & $2(100)$ & 0 & $6(100)$ & $1(100)$ \\
\hline AVA positive & 0 & 0 & 0 & 0 \\
\hline \multicolumn{5}{|l|}{ No } \\
\hline No. & 25 & 10 & 4 & 1 \\
\hline AVA negative & $23(92)$ & $7(70)$ & $3(75)$ & $1(100)$ \\
\hline AVA positive & $2(8)$ & $3(30)$ & $1(25)$ & 0 \\
\hline
\end{tabular}

Values are presented as number (\%).

AVA, anti-vedolizumab antibody; IV, intravenous; SC, subcutaneous. 